\title{
Improvement of Cylindrical Cloak by Layered Structure of Homogeneous Isotropic Materials
}

\author{
Jun DING, Yang LIU, Chenjiang GUO, Qian XU
}

School of Electronic and Information Engineering, Northwest Polytechnical University, Xi'an, China.

Email: dingjun@nwpu.edu.cn

Received September $8^{\text {th }}, 2009$; revised September $26^{\text {th }}, 2009$; accepted September $29^{\text {th }}, 2009$.

\begin{abstract}
The perfect cylindrical cloak requires three spatial variant material parameters, which is very difficult to realize in practice [Science 312, 1780 (2006)]. The approach of realizing the electromagnetic cloaking by concentric layered structures instead of using the metamaterial was presented [Optics Express, Vol. 15, No. 18 (2007)]. We use the concentric layered structures to realize a simplified cylindrical cloak with improved parameters and an ideal cylindrical cloak with spatially invariant axial material parameters. Numerical simulation results validate that cloaking performance is significantly improved compared with previously proposed multilayered cloak.
\end{abstract}

Keywords: Cylindrical Cloak, Concentric Layered Structures

\section{Introduction}

Recently the idea of designing an invisible cloak by means of coordinate transformation approach has drawn extensive attention [1-23]. Pendry et al. [1] first suggested an interesting way of using the coordinate transformation method to design a cloak of invisibility. Many theoretical studies have shown the possibility of such invisible cloaks $[2,3]$. The first experimental demonstration of such invisible cloak using metamaterial with simplified material parameters was soon realized [4], the simplified cloak inherits some properties of the ideal cloak, but finite scatterings exist. Perfect electromagnetic invisible cloak requires inhomogeneous and anisotropic media, therefore it is difficult to construct by using naturally existed materials. A cylindrical cloak structure that does not require metamaterials to realize the anisotropy and inhomogeneity of the material parameters was proposed [9], it was realized by a concentric layered structure consisting of alternating homogeneous isotropic materials, which can be treated as an effective medium with the radius dependent anisotropy.

In this paper, we use the concentric layered structures to construct a simplified cylindrical cloak with improved parameters and an ideal cylindrical cloak with spatially invariant axial material parameters. It may be realized by layered structures of composite media.

\section{Cylindrical Cloak by Layered Structure of Homogeneous Isotropic Materials}

Cylindrical anisotropy can be attained by layered struc- ture of homogeneous isotropic materials $[10,11]$. As shown in Figure 1, we assume that each layer can be described by homogeneous and isotropic permittivity and permeability parameters. The layer thickness $d$ in each of these structures is much less than the wavelength $\lambda$ and when $d \ll \lambda$, we can treat the structured material as a single anisotropic medium with dielectric permittivity

$$
\varepsilon_{r}=\frac{(1+\eta) \varepsilon_{1} \varepsilon_{2}}{\varepsilon_{2}+\eta \varepsilon_{1}}, \quad \varepsilon_{\theta}=\frac{\varepsilon_{1}+\eta \varepsilon_{2}}{1+\eta}
$$

where $\eta=d_{2} / d_{1}$ is the thicknesses ratio of the two layers.

As proposed in Reference [9], the cylindrical invisible with inhomogeneous and anisotropic media can be con

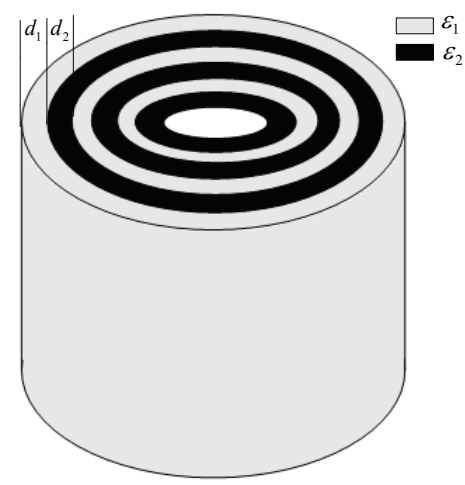

Figure 1. Concentric layers structure alternate with dielectric layers 
structed through layered structures of homogeneous isotropic materials. Now we concentrate on the realization of the improved simplified cloak and the ideal cylindrical cloak through multilayered structures of composite materials.

As proposed in Reference [16], we could obtain another set of cloak parameters satisfying the requirement, the associate material parameters of the cloak can be expressed as

$$
\varepsilon_{r}=\frac{b}{b-a}\left(\frac{r-a}{r}\right)^{2}, \quad \varepsilon_{\theta}=\frac{b}{b-a}, \quad \mu_{z}=\frac{b}{b-a}
$$

We first apply this idea to construct a cylindrical cloak with improved parameters, combining Equations (1) and (2), only $\varepsilon_{r}, \varepsilon_{\theta}$ are considered and $\mu_{z}$ is invariant, we obtain the expressions:

$$
\begin{array}{r}
\frac{b}{b-a}\left(\frac{r-a}{r}\right)^{2}=\frac{(1+\eta) \varepsilon_{1} \varepsilon_{2}}{\varepsilon_{2}+\eta \varepsilon_{1}} \\
\frac{b}{b-a}=\frac{\varepsilon_{1}+\eta \varepsilon_{2}}{1+\eta}
\end{array}
$$

There are two ways to achieve the multilayered cylindrical cloak. One is to keep the thickness ratio $\eta$ a constant value and alternate layers of isotropic media $\varepsilon_{1}$ and $\varepsilon_{2}$ vary with the radius. The other way is to construct a concentric layered structure that the permittivity of the alternating dielectrics is fixed, while the thickness ratio of the two layers varies to approximately satisfy the Equation (3).

Here, we take the fist approach as an example, assume the thickness of the two layers are equal $(\eta=1)$, the cylindrical cloaking shell with inner radius $a=\lambda$ and outer radius $b=2 \lambda$, then Equation (3) can be written in the form:

$$
\varepsilon_{1}+\varepsilon_{2}=4, \quad \varepsilon_{1} \varepsilon_{2}=4\left(\frac{r-a}{r}\right)^{2}
$$

Next, we consider a stepwise homogeneous ten discrete layer approximation of the ideal, continuous parameters required by Equation (4), which has removed the most challenging issue of the design, the continuous radius-dependent, anisotropic medium could be represented approximately by ten discrete layers of homogeneous anisotropic medium. Then we use the alternating layers of isotropic dielectric $\varepsilon_{1}$ and $\varepsilon_{2}$ to mimic each homogeneous anisotropic layer, the permitivities of them are designed by Equation (4) according to the corresponding anisotropic layer. The thinner the layers are, the better the layered structure approaches the ideal anisotropic medium. We can conclude the dielectric $\varepsilon_{1}$ and $\varepsilon_{2}$ in each discrete layer by Equation (4), as shown in Table 1 .
The relative permittivity of anisotropic medium varies gradually, dielectric $\varepsilon_{1}$ increases gradually from 0.008 to 0.268 , while the relative permittivity of dielectric $\varepsilon_{2}$ decreases gradually from 3.992 to 3.372 . Here, we should note that the relative permeability of the layers is 2 , which may be realized by composite media. Now, we have completed the material parameters description.

Following the above approach, we can easily realize the ideal cylindrical cloak through multilayered structures of composite materials. Yu Luo et al. [17] have provided a perfect cylindrical invisible cloak with spatially invariant axial material parameters:

$$
\varepsilon_{r}=\frac{r^{2}-a^{2}}{r^{2}}, \quad \varepsilon_{\theta}=\frac{r^{2}}{r^{2}-a^{2}}, \quad \mu_{z}=\frac{b^{2}}{b^{2}-a^{2}}
$$

Combining Equations (1) and (5), we obtain the expressions:

$$
\begin{aligned}
\frac{r^{2}-a^{2}}{r^{2}} & =\frac{(1+\eta) \varepsilon_{1} \varepsilon_{2}}{\varepsilon_{2}+\eta \varepsilon_{1}} \\
\frac{r^{2}}{r^{2}-a^{2}} & =\frac{\varepsilon_{1}+\eta \varepsilon_{2}}{1+\eta}
\end{aligned}
$$

Plugging in the cloak parameters value, we find that the media properties are given by

$$
\varepsilon_{1} \varepsilon_{2}=1, \varepsilon_{1}+\varepsilon_{2}=\frac{2 r^{2}}{r^{2}-a^{2}}
$$

We can also conclude the dielectric $\varepsilon_{1}$ and $\varepsilon_{2}$ of each discrete layer in multilayered cloak with perfect

Table 1. The permittivity of ten-layered multilayered cloak with improved simplified parameters

\begin{tabular}{cccccc}
\hline $\begin{array}{c}\text { Layer } \\
\text { (unit: } \lambda \text { ) }\end{array}$ & $1.0 \sim 1.1$ & $1.1 \sim 1.2$ & $1.2 \sim 1.3$ & $1.3 \sim 1.4$ & $1.4 \sim 1.5$ \\
\hline$\varepsilon_{1}$ & 0.008 & 0.028 & 0.054 & 0.083 & 0.114 \\
$\varepsilon_{2}$ & 3.992 & 3.972 & 3.946 & 3.917 & 3.886 \\
$\begin{array}{c}\text { Layer } \\
\text { (unit: } \lambda \text { ) }\end{array}$ & $1.5 \sim 1.6$ & $1.6 \sim 1.7$ & $1.7 \sim 1.8$ & $1.8 \sim 1.9$ & $1.9 \sim 2.0$ \\
$\varepsilon_{1}$ & 0.146 & 0.177 & 0.208 & 0.239 & 0.268 \\
$\varepsilon_{2}$ & 3.854 & 3.823 & 3.792 & 3.761 & 3.732 \\
\hline
\end{tabular}

Table 2. The permittivity of ten-layered multilayered cloak with perfect parameters

\begin{tabular}{cccccc}
\hline $\begin{array}{c}\text { Layer } \\
\text { (unit: } \lambda \text { ) }\end{array}$ & $1.0 \sim 1.1$ & $1.1 \sim 1.2$ & $1.2 \sim 1.3$ & $1.3 \sim 1.4$ & $1.4 \sim 1.5$ \\
\hline$\varepsilon_{1}$ & 0.0874 & 0.1565 & 0.2134 & 0.2617 & 0.3033 \\
$\varepsilon_{2}$ & 11.4364 & 6.3889 & 4.6851 & 3.8217 & 3.2967 \\
$\begin{array}{c}\text { Layer } \\
\text { (unit: } \lambda \text { ) }\end{array}$ & $1.5 \sim 1.6$ & $1.6 \sim 1.7$ & $1.7 \sim 1.8$ & $1.8 \sim 1.9$ & $1.9 \sim 2.0$ \\
$\varepsilon_{1}$ & 0.3399 & 0.3723 & 0.4014 & 0.4276 & 0.4514 \\
$\varepsilon_{2}$ & 2.9422 & 2.6859 & 2.4915 & 2.3387 & 2.2153 \\
\hline
\end{tabular}


material parameters by Equation (7), as shown in Table 2, here, we note that $\mu=4 / 3$.

Full-wave simulations with the commercial finite-element solver COMSOL MULTIPHYSICS were performed to verify the performance of the designed electromagnetic cloak. In the simulations, we assume a TM plane wave incidence along the $x$ direction. The cloaking materials are assumed to be lossless and the interior region is perfect electric conductor (PEC) ensuring that the fields inside it are ideally zero, and also demonstrating the cloaking performance.

Perfect matched layers(PML) are used to absorb the scattered field, which simulate the infinite domain in which the system resides, and 10 sub-layers of alternat- ing permittivity are used to realize each layer. The simulated fields shown in each case were computed with approximately 170,000 elements and 200,000 unknowns.

Figure 2(a) shows the simulation results for the magnetic field and scattered magnetic field distribution around the multilayered cylindrical cloak with improved parameters, the magnetic fields are smoothly excluded from the interior region with minimal scattering. The scattered fields are caused by the reduced set of parame-ters and small approximation made in the design of the permittivity and the thickness ratio. Figure 2(b) suggests that the multilayered cylindrical cloak with perfect mate rial parameters should cause a relatively low scattering at the same cloak thicknesses. The scattered fields

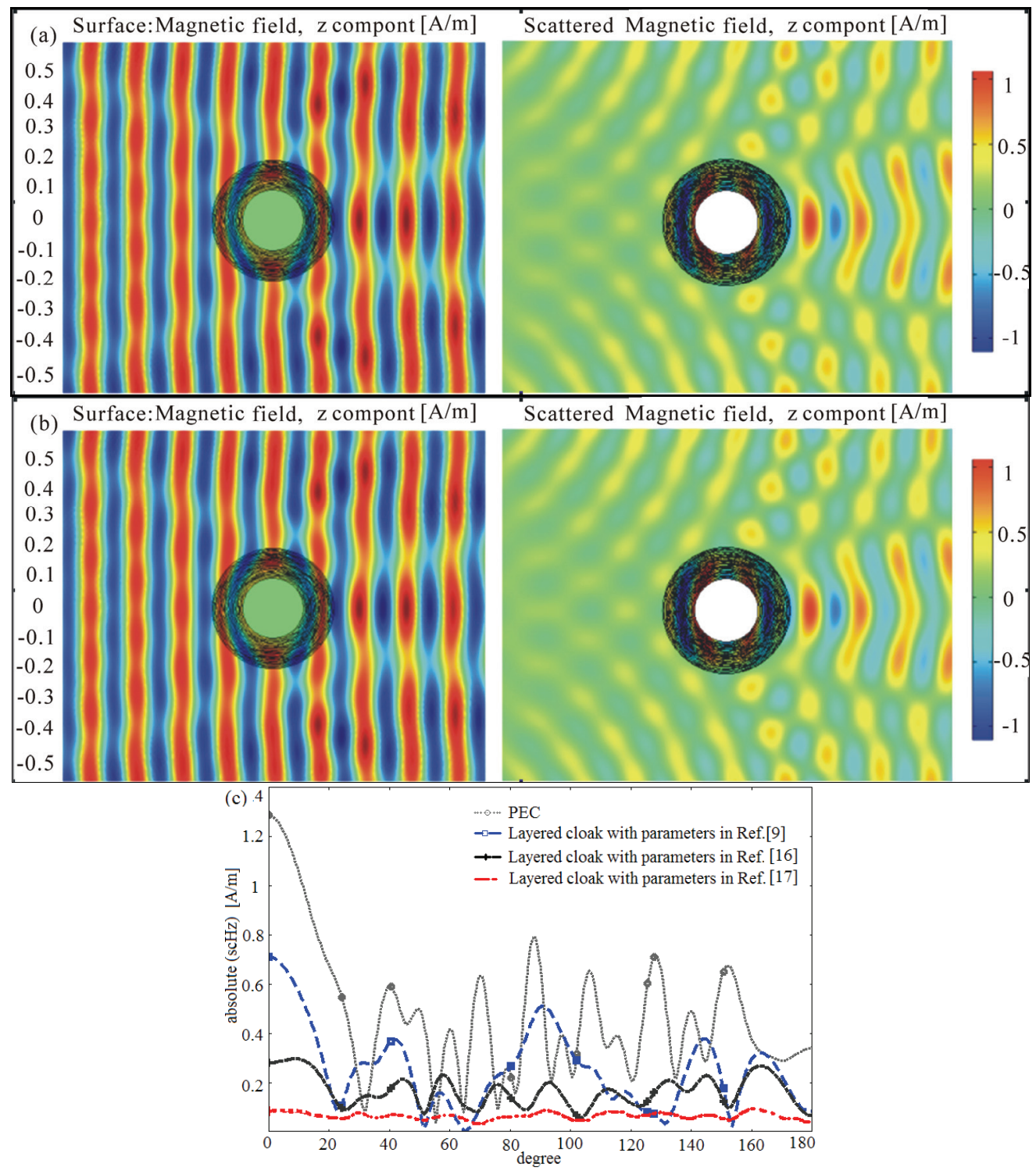

Figure 2. The magnetic-field and scattered magnetic field distribution around the electromagnetic cloak, (a) a concentric multilayered cylindrical cloak with improved simplified parameters, (b) a multilayered cylindrical cloak with perfect parameters, (c) comparison of absolute scattered magnetic field of $\mathrm{z}$ component 
are caused by the approximations made in the design of the permittivity and the thickness ratio. As mentioned above, the nonideal cylindrical cloak by layered structure of homogeneous isotropic media has been already discussed in [9]. We explained that the difference is that we are constructing a multilayered cylindrical cloak with improved parameters and a multilayered cloak with ideal parameters by the concentric layered structures. We have also compared the scattering characteristics of three types of cylindrical cloak (Figure 2(c)). It shows clearly that both multilayered cloak with improved simplified parameters and ideal material parameters have smaller scattering, compared with previously reported layered cloak [9].

\section{Conclusions}

In conclusion, we construct a cylindrical cloak with improved simplified parameters and an ideal cylindrical cloak by using spatially invariant axial material parameters through layered structures of alternating homogeneous isotropic materials. These two cloaks exhibit better invisibility performances as compared to the previously reported cloak. In our design, the relative permeability of material media is always larger than 1 , which may be realized by composite media. Such cloak has no requirement of any metamaterial, it is possible to be realized by thin layers of composite media. With its relatively low requirement on material parameters, it can potentially be a better candidate for realizing a near-invisible cloak.

\section{Acknowledgment}

This work was supported by graduate starting seed fund of Northwestern Polytechnical University.

\section{REFERENCES}

[1] J. B. Pendry, D. Schurig, and D. R. Smith, "Controlling electromagnetic fields," Science 312, 1780, 2006.

[2] J. B. Pendry, D. Schurig, and D. R. Smith, "Calculation of material properties and ray tracing in transformation media," Optics Express 14, 9794, 2006.

[3] S. Cummer, B.-I. Popa, D. Schurig, D. Smith, and J. Pendry, "Full wave simulations of electromagnetic cloaking structures," Physical Review E74, 036621, 2006.

[4] D. Schurig, J. J. Mock, B.-J. Justice, S. A. Cummer, J. B. Pendry, A. F. Starr, and D. R. Smith, "Metamaterial electromagnetic cloak at microwave frequencies," Science, 2006.

[5] W. S. Cai, U. K. Chettiar, A. V. Kildishev, and V. M. Shalaev, "Optical cloaking with metamaterials," Nature Photonics, Vol. 1, pp. 224-27, 2007.

[6] W. S. Cai, U. K. Chettiar, A. V. Kildishev, G. W. Milton, and V. M. Shalaev, "Nonmagnetic cloak with minimized scattering," Appled Physical Letters 91, 111105, 2007.

[7] W. S. Cai, U. K. Chettiar, A. V. Kildishev, and V. M. Shalaev, "Designs for optical cloaking with high-order transformations," Optics Express 5444, Vol. 16, No. 8, 2008.
[8] M. Rahm, D. Schurig, D. A. Roberts, S. A. Cummer, D. R. Smith, and J. B. Pendry, "Design of electromagnetic cloaks and concentrators using form-invariant coordinate transformations of Maxwell's equations," Photon Nanostructured: Fundamental Application, Vol. 6, pp. 87-95, 2008 .

[9] Y.Huang, Y. J. Feng, T. Jiang, "Electromagnetic cloaking by layered structure of homogeneous isotropic materials," Optics Express, Vol. 15, pp. 11133-11141, 2007.

[10] Z. Jacob, L. V. Alekseyev, E. Narimanov, "Optical hyperlens: Far-field imaging beyond the diffraction limit," Optics Express, Vol. 14, pp. 8247-8256, 2006.

[11] A. Salandrino and N. Engheta, "Far-field subdiffraction optical microscopy using metamaterial crystals: Theory and simulations", Physical Review, B74, 075103, 2006.

[12] H. S. Chen, B.-I. Wu, B. L. Zhang, and J. A. Kong, "Electromagnetic wave interactions with a metamaterial cloak," PRL99, 063903, 2007.

[13] B. L. Zhang, H. S. Chen, B.-I. Wu, Y. Luo, L. X. Ran, and J. A. Kong, "Response of a cylindrical invisibility cloak to electromagnetic waves," Physical Review, B76, 121101, 2007.

[14] Z. C. Ruan, M, Yan, C, W. Neff, and M, Qiu, "Ideal cylindrical cloak: Perfect but sensitive to tiny perturbations," Physical Review Letters, 99, 113, 903, 2007.

[15] M. Yan, Z. Ruan, and M. Qiu, "Cylindrical invisibility cloak with simplified material parameters is inherently visible," Physical Review Letters 99, 233, 901, 2007.

[16] M. Yan, Z. C. Ruan, and M. Qiu, "Scattering characteristics of simplified cylindrical invisibility cloaks," Optics Experss17772, Vol. 15, No. 26, 2007.

[17] Y. Luo, J. J. Zhang, H. S. Chen, a- S. Xi, and B.-I. Wu, "Cylindrical cloak with axial permittivity/permeability spatially invariant," Applied Physics Letters 93, 033504, 2008.

[18] G. Isí, R. Gajić, B. Novaković, Z. V. Popović, and K. Hingerl, "Radiation and scattering from imperfect cylindrical electromagnetic cloaks," Optics Express 1413, Vol. 16, No. 3, 2008

[19] J. J. Zhang, Y. Luo, H. S. Chen, B.-I. Wu, "Cloak of arbitrary shape", Journal of the Optical Society of America B 25, pp. 1776-1779, 2008.

[20] J. J. Zhang, J. T. Huang, Y. Luo, H. S. Chen, J. A. Kong, and B.-I. Wu. "Cloak for multilayered and gradually changing media," Physical Review B 77, 035116, 2008.

[21] Y. Luo, et al., "Design and analytical full-wave validation of the invisibility cloaks, concentrators, and field rotators created with a general class of transformations", Physical Review B, 77, 125127, 2008.

[22] J. A. Silva-Maćedo, M. A. Romero, and B.-H. V. Borges, "An extended FDTD method for the analysis of electromagnetic field rotations and cloaking devices," Progress In Electromagnetics Research, PIER 87, pp. 183-196, 2008.

[23] J. J. Zhang, Y. Luo, H. S. Chen, and B.-I. Wu, "Sensitivity of transformation cloak in engineering," Progress In Electromagnetics Research, PIER 84, pp. 93-104, 2008. 